\title{
Mixed-mode stress intensity factors evaluation of flat shells under in-plane loading employing ordinary state-based peridynamics
}

\author{
Ming-Jyun Dai ${ }^{\mathrm{a}}$, Satoyuki Tanaka ${ }^{\mathrm{a}, *}$, Selda Oterkus ${ }^{\mathrm{b}}$, Erkan Oterkus $^{\mathrm{b}}$ \\ ${ }^{a}$ Graduate School of Advanced Science and Engineering, \\ Hiroshima University, Japan \\ ${ }^{b}$ Department of Naval Architecture, Ocean and Marine Engineering, \\ University of Strathclyde, United Kingdom
}

\begin{abstract}
A recently developed peridynamic (PD) shell model based on the ordinary state-based peridynamic theory is adopted to evaluate stress intensity factors (SIFs) under in-plane loading. Strain and stress components are obtained by introducing the peridynamic differential operator. In order to evaluate mixed-mode SIFs, the domain form of the interaction integral is employed. The adaptive dynamic relaxation technique is utilized to obtain steady-state solutions, and the energy method is applied to reduce the PD surface effect. Several numerical examples are considered, including single- and mixed-mode fracture problems. All the PD results are compared with reference results to demonstrate the accuracy and effectiveness of the proposed approach. The present paper aims to examine the performance of the PD shell model in linear elastic fracture mechanics and provides an effective approach for SIFs evaluation.
\end{abstract}

Keywords: Peridynamics; Peridynamic differential operator; Interaction integral; Stress intensity factors; Fracture; Flat shell

\section{Introduction}

Structural failure prediction is a challenging work in computational mechanics. In classical continuum mechanics (CCM), the governing equations

\footnotetext{
${ }^{*}$ Corresponding author. E-mail addresses: satoyuki@hiroshima-u.ac.jp
} 
are based on partial differential equations (PDEs). PDEs, however, are not suited for modeling cracks owing to displacement discontinuities. Therefore, some special numerical techniques are necessarily proposed in conventional numerical methods. Peridynamics (PD) which is a non-local continuum theory had been introduced by Silling [1,2]. The PD formulations are based on integro-differential equations which can naturally describe discontinuities in the analysis domain without any special treatments.

Most PD studies concentrate on the two-dimensional (2D) plane and three-dimensional (3D) solid models. However, simulating a thin-walled structure by the 3D PD model is computationally expensive. Structural idealization is one of the approaches to reduce computational time. In CCM, the Kirchhoff-Love and Mindlin-Reissner plate theories are common approaches of structural idealization for thin-walled structures. Structural idealization is also a research branch in the PD theory. O'Grady and Foster [3] introduced a rotational spring between bonds to simulate the Kirchhoff-Love plate by nonordinary state-based peridynamics (NOSPD). Diyaroglu et al. [4] developed a Mindlin plate model by bond-based peridynamics (BBPD). Chowdhury et al. [5] proposed a curved bond for linear elastic shells by NOSPD. Nguyen and Oterkus [6] developed a 3D shell PD model based on the Mindlin plate theory with drilling rotation by ordinary state-based peridynamics (OSPD). Yang et al. [7] introduced a Kirchhoff plate model and imposed various boundary conditions (BCs) by OSPD. Nguyen and Oterkus [8] applied a 3D shell PD model to evaluate the strength of maritime structures.

Several criteria in the PD theory can be found in previous studies. First, the critical stretch based on bond elongation [9] was proposed. The critical stretch mainly deals with mode-I brittle fracture problems. Hence, the energy-based failure criteria $[10,11]$ were subsequently introduced. Dipasquale et al. [12] compared the existing failure criteria in mixed-mode brittle fracture problems and concluded that a more reliable failure criterion is required for complex fracture problems.

Critical fracture toughness $\left(K_{c}\right)$ is the major failure criterion in most experimental and numerical analyses. If stress intensity factor (SIF) in a cracked structure reaches its critical value, $K_{c}$, crack propagation will take place. In the PD literature, SIFs investigations with respect to the foundation of classical fracture mechanics are rare. Hu et al. [13] derived the peridynamic J-integral and the results are compared with FEM. Breitenfeld et al. [14] examined the accuracy of field variables in the vicinity of the crack front. Le and Bobaru [15] carried out the PD J-integral evaluation with 
the different techniques of surface effect correction. Stenström and Eriksson [16] presented the J-integral formulation via displacements from BBPD. The studies mentioned above only considered pure mode-I fracture problems. Few papers discussed mixed-mode cases in the PD framework. Jung and Seok [17] decomposed the PD J-integral into mode-I and -II SIFs to implement fatigue crack growth analysis. Imachi et al. [18] evaluated mixed-mode dynamic SIFs for 2D elastic cracked solids.

In the present paper, we provide an approach to evaluate mixed-mode SIFs in the PD framework. The OSPD model for shell structures [6] is employed to deal with fracture problems. The energy method [19] is applied to reduce the surface effect which affects the accuracy of results near domain boundaries. To obtain steady-state solutions, the adaptive dynamic relaxation (ADR) technique [19] is introduced. Additionally, the derivative of variables in the J-integral formulation is evaluated by introducing the peridynamic differential operator (PDDO) [20]. The interaction integral [21] is adopted to obtain mixed-mode SIFs. From the conclusions presented in Ref. [22], membrane SIFs are generally greater than bending SIFs in thin cracked plates. In thin-walled structures, most of the loads are transferred to the structure as in-plane stresses $[22,23]$. Thus, in-plane loads are considered throughout the present paper.

The remainder of the paper is organized as follows. The PD shell model is briefly introduced in Section 2. The PDDO and interaction integral are presented to evaluate mixed-mode SIFs in Section 3. Four numerical examples are shown in Section 4. The conclusions are given in Section 5.

\section{Ordinary state-based peridynamic formulation}

\subsection{Peridynamic model for shell structures}

The PD model for shell structures is based on the ordinary state-based PD theory. In CCM, the state of a material point is influenced by the material points located in its immediate vicinity. In contrast to CCM, any point $\boldsymbol{x}$ interacts with the other material points within a distance $\delta$ in the PD theory. The non-local region is called the horizon. The material points within $\delta$ are called the family of $\boldsymbol{x}, H_{\boldsymbol{x}}$ [19]. In Fig. 1, the interactional relationship of material points between CCM and PD is illustrated.

First, the strain energy densities of the PD shell model are defined, including in-plane, bending, and shear terms. The strain energy densities are 
represented in a similar manner to the Mindlin plate theory. Next, the equations of motion of this PD shell model are derived from the principle of virtual work. Here, the PD shell model is briefly introduced. The details can be found in Ref. [6]. Note that the small deformation assumption is taken in the proposed PD model.

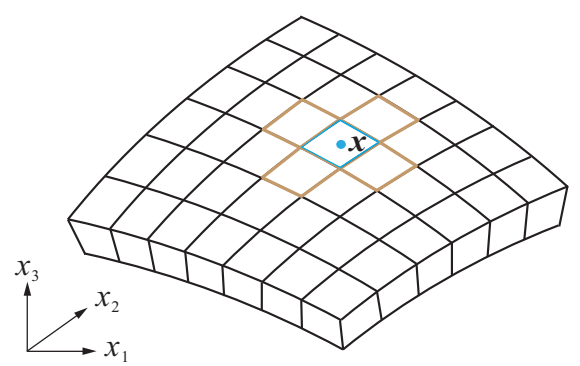

Classical continuum mechanics

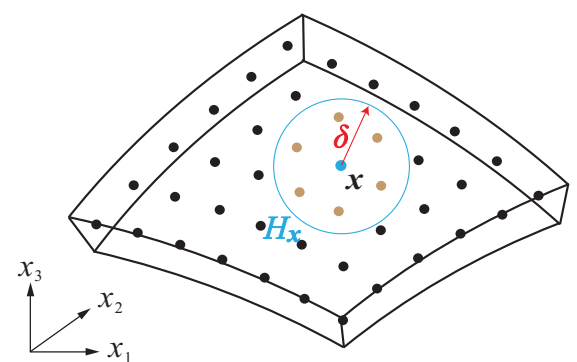

Peridynamics

Figure 1: The interactional relationship of material points between CCM and PD.

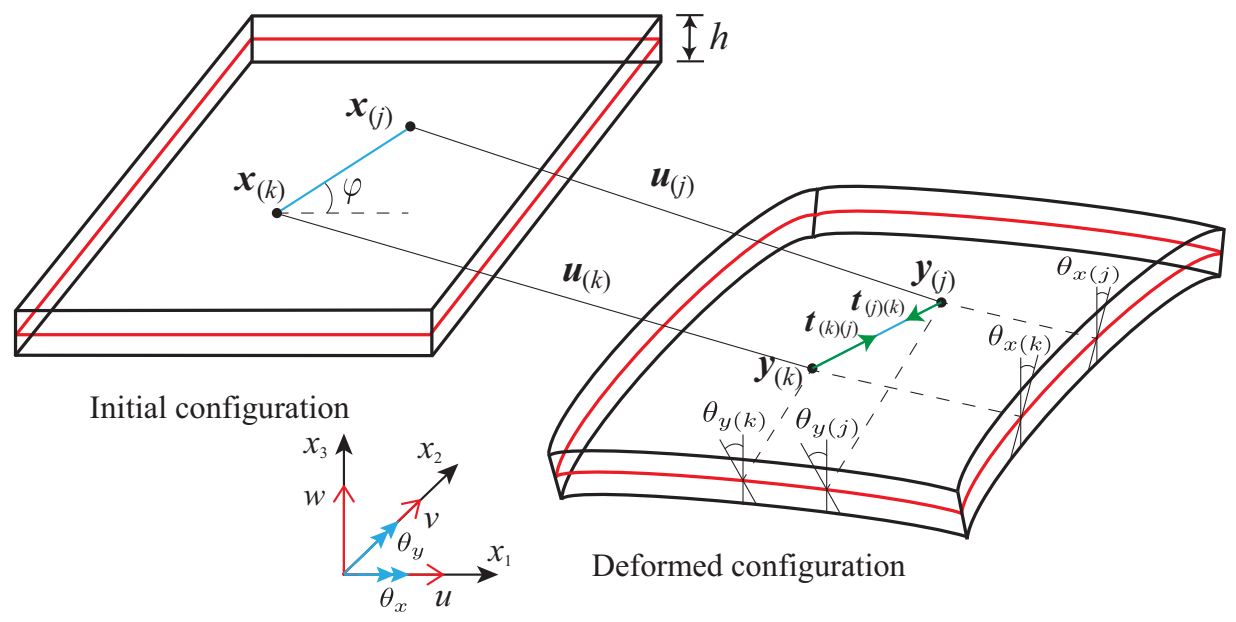

Figure 2: Initial and deformed states of a flat shell in PD.

At any instant of time, the material point $k$ interacts with the other material points $j$ in its own family, $H_{x}$. The interaction between points $k$ and $j$ is presented in Fig. 2. $\boldsymbol{x}_{(k)}$ and $\boldsymbol{y}_{(k)}$ denote the initial and deformed positions 
of point $k$, respectively. $\varphi$ represents the angle of interaction between points $k$ and $j$ with respect to the $x$-axis. $\boldsymbol{u}_{(k)}=\left[\begin{array}{llll}u & v & w & \theta_{x} \\ \theta_{y}\end{array}\right]^{T}$ and $\boldsymbol{t}_{(k)(j)}$ denote the displacement and force density vectors of point $k$, respectively. The equation of motion for the PD shell model can be written in vector form as:

$$
\boldsymbol{m}_{(k)} \ddot{\boldsymbol{u}}_{(k)}=\sum_{j=1}^{N}\left(\boldsymbol{t}_{(k)(j)}-\boldsymbol{t}_{(j)(k)}\right) V_{(j)}+\boldsymbol{b}_{(k)}=\sum_{j=1}^{N} \boldsymbol{f}_{(k)(j)} V_{(j)}+\boldsymbol{b}_{(k)},
$$

where $N$ is the total number of material points within $\delta$, and $V_{(j)}$ is the volume of point $j$. Mass matrix $\boldsymbol{m}_{(k)}$, acceleration vector $\ddot{\boldsymbol{u}}_{(k)}$, force density vector $\boldsymbol{f}_{(k)(j)}$, and body force density vector $\boldsymbol{b}_{(k)}$ are given as below:

$$
\boldsymbol{m}_{(k)}=\left[\begin{array}{ccccc}
\rho h & 0 & 0 & 0 & 0 \\
0 & \rho h & 0 & 0 & 0 \\
0 & 0 & \rho h & 0 & 0 \\
0 & 0 & 0 & \frac{\rho h^{3}}{12} & 0 \\
0 & 0 & 0 & 0 & \frac{\rho h^{3}}{12}
\end{array}\right]
$$

where $\rho$ and $h$ represent the mass density and thickness of the flat shell, respectively.

$$
\ddot{\boldsymbol{u}}_{(k)}=\left[\begin{array}{c}
\ddot{u}_{(k)} \\
\ddot{v}_{(k)} \\
\ddot{w}_{(k)} \\
\ddot{\theta}_{x(k)} \\
\ddot{\theta}_{y(k)}
\end{array}\right], \quad \boldsymbol{f}_{(k)(j)}=\left[\begin{array}{c}
f_{(k)(j)}^{u} \\
f_{(k)(j)}^{v} \\
f_{(k)(j)}^{w} \\
f_{(k)(j)}^{\theta_{x}} \\
f_{(k)(j)}^{\theta_{y}}
\end{array}\right], \quad \boldsymbol{b}_{(k)}=\left[\begin{array}{c}
b_{x(k)} \\
b_{y(k)} \\
b_{z(k)} \\
m_{x(k)} \\
m_{y(k)}
\end{array}\right] .
$$

The force density vector $\boldsymbol{f}_{(k)(j)}$ of each component is constructed by PD parameters, bond stretch $s$, dilatation $\vartheta$, and displacements $\boldsymbol{u}$. The PD force densities are expressed as below:

$$
\begin{aligned}
& f_{(k)(j)}^{u}=\left[\frac{2 a_{i p} d_{i p}}{\xi}\left(\vartheta_{i p(k)}+\vartheta_{i p(j)}\right)+4 b_{i p} s_{i p(k)(j)}\right] \cos \varphi, \\
& f_{(k)(j)}^{v}=\left[\frac{2 a_{i p} d_{i p}}{\xi}\left(\vartheta_{i p(k)}+\vartheta_{i p(j)}\right)+4 b_{i p} s_{i p(k)(j)}\right] \sin \varphi,
\end{aligned}
$$




$$
\begin{aligned}
f_{(k)(j)}^{w}= & C_{s}\left\{\frac{w_{(j)}-w_{(k)}}{\xi}-\frac{1}{2}\left[-\left(\theta_{y(k)}+\theta_{y(j)}\right) \cos \varphi\right.\right. \\
& \left.\left.+\left(\theta_{x(k)}+\theta_{x(j)}\right) \sin \varphi\right]\right\}, \\
f_{(k)(j)}^{\theta_{x}}=\left[\frac{2 a_{b} d_{b}}{\xi}\left(\vartheta_{b(k)}+\vartheta_{b(j)}\right)+4 b_{b} s_{b(k)(j)}\right] \sin \varphi & \\
+ & \frac{1}{2} C_{s}\left\{\left(w_{(j)}-w_{(k)}\right) \sin \varphi\right. \\
& \left.-\frac{\xi}{2}\left[-\left(\theta_{y(k)}+\theta_{y(j)}\right) \sin \varphi \cos \varphi+\left(\theta_{x(k)}+\theta_{x(j)}\right) \sin ^{2} \varphi\right]\right\}, \\
f_{(k)(j)}^{\theta_{y}}= & -\left[\frac{2 a_{b} d_{b}}{\xi}\left(\vartheta_{b(k)}+\vartheta_{b(j)}\right)+4 b_{b} s_{b(k)(j)}\right] \cos \varphi \\
& -\frac{1}{2} C_{s}\left\{\left(w_{(j)}-w_{(k)}\right) \cos \varphi\right. \\
& \left.-\frac{\xi}{2}\left[-\left(\theta_{y(k)}+\theta_{y(j)}\right) \cos { }^{2} \varphi+\left(\theta_{x(k)}+\theta_{x(j)}\right) \sin \varphi \cos \varphi\right]\right\} .
\end{aligned}
$$

The dilatation $\vartheta_{l(k)}$ is the relative variation of volume at a material point. In the PD theory, the dilatation is a function of bond stretch $s_{l(k)(j)} . \quad l=$ ip and $l=\mathrm{b}$ represent the dilatation for in-plane and bending deformations, respectively. The dilatation is defined as:

$$
\vartheta_{l(k)}=d_{l} \sum_{j=1}^{N} s_{l(k)(j)} V_{(j)} .
$$

The bond stretch for in-plane deformation $s_{i p(k)(j)}$ and bending deformation $s_{b(k)(j)}$ are given below:

$$
\begin{gathered}
s_{i p(k)(j)}=\frac{\left(u_{(j)}-u_{(k)}\right) \cos \varphi+\left(v_{(j)}-v_{(k)}\right) \sin \varphi}{\xi}, \\
s_{b(k)(j)}=\frac{-\left(\theta_{y(j)}-\theta_{y(k)}\right) \cos \varphi+\left(\theta_{x(j)}-\theta_{x(k)}\right) \sin \varphi}{\xi},
\end{gathered}
$$


where $\xi$ is the distance between $\boldsymbol{x}_{(k)}$ and $\boldsymbol{x}_{(j)}$.

The PD parameters can be derived by comparing dilatations and strain energy densities between CCM and PD. The subscripts of $i p, b$, and $s$ in the PD parameters represent in-plane, bending, and shear terms, respectively. The PD parameters are presented as:

$$
\begin{gathered}
a_{i p}=\frac{E h(3 \nu-1)}{4\left(1-\nu^{2}\right)}, \quad d_{i p}=\frac{3 E}{\pi \delta^{3}(1+\nu)}, \quad b_{i p}=\frac{2}{\pi h \delta^{2}}, \\
a_{b}=\frac{E h^{3}(3 \nu-1)}{48\left(1-\nu^{2}\right)}, \quad d_{b}=\frac{E h^{2}}{4 \pi \delta^{3}(1+\nu)}, \quad b_{b}=\frac{2}{\pi h \delta^{2}}, \\
C_{s}=\frac{3 k_{s} E}{\pi \delta^{3}(1+\nu)},
\end{gathered}
$$

where $\delta, E, \nu$, and $k_{s}$ denote the horizon size, Young's modulus, Poisson's ratio, and shear correction factor, respectively.

\subsection{Numerical implementation and crack modeling}

The equations of motion in $\mathrm{PD}$ can directly solve dynamic problems. For static and quasi-static problems, the ADR technique [19] is necessarily adopted in PD solutions. By replacing the mass matrix with the stable mass matrix and damping matrix into the equations of motion, the steady-state solution can be obtained to solve static problems by the ADR method. The stable mass matrix for the PD shell model is introduced in Ref. [6].

It is assumed that the PD parameters are computed under complete neighborhood volumes in the horizon. However, the horizon does not completely embed in the analysis domain while $\boldsymbol{x}$ approaches domain boundaries. The parameter value with the complete horizon is slightly different from the value without the complete horizon [15]. This is called the "surface effect" in the PD theory. The energy method [19] is proposed to reduce the surface effect and obtain more accurate results.

The analysis domain is discretized by uniform scattered particles. A crack is modeled on the analysis domain by disconnecting bonds between material points. When the crack segment intersects with the interaction bond, the interaction bond is irreversibly broken. Crack modeling in the PD 


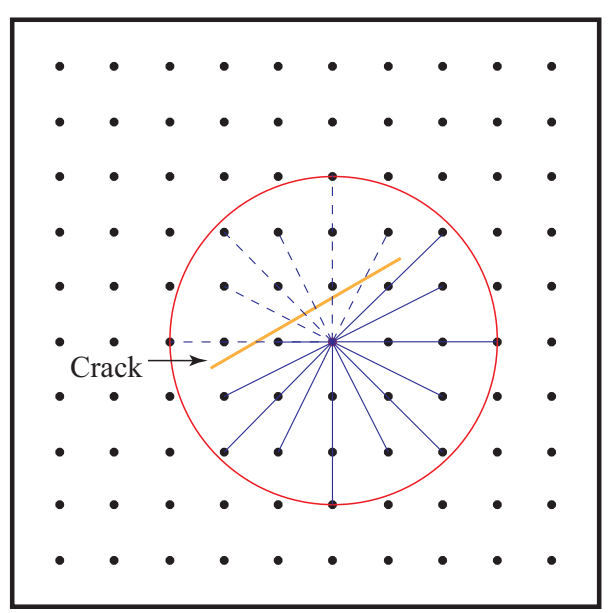

Figure 3: Schematic of crack modeling in PD.

theory is expressed in Fig. 3. The solid lines and dashed lines represent the connected bonds and broken bonds, respectively. The state of bond interaction is represented by $\mu(\boldsymbol{\xi}, t)$ in Eq. (15) [9].

$$
\mu(\boldsymbol{\xi}, t)= \begin{cases}0 & \text { broken bond } \\ 1 & \text { intact bond }\end{cases}
$$

\section{Stress intensity factors evaluation}

\subsection{Peridynamic differential operator}

Generally, the PD theory does not concern derivatives of physical variables, such as strain and stress. However, strain and stress are possible to be utilized during numerical implementation in the PD framework, especially for SIFs evaluation. The PD stress tensor based on nonlocal interactions was derived by Silling and Lehoucq [24]. Breitenfeld et al. [14] developed the nonlocal deformation gradient to obtain strain and stress tensors in the NOSPD formulation. Subsequently, the PD differential operator is proposed in the concept of nonlocal interactions by Madenci et al. [20]. The PDDO can deal with higher-order derivatives without any limitations. Thus, the PDDO is adopted to evaluate strain and stress components in the present 
paper. Field variables can be constructed by the Taylor series expansion. The 2D spatial function can be written as:

$$
\begin{aligned}
f(\boldsymbol{x}+\boldsymbol{\xi})= & f(\boldsymbol{x})+\xi_{1} \frac{\partial f(\boldsymbol{x})}{\partial x_{1}}+\xi_{2} \frac{\partial f(\boldsymbol{x})}{\partial x_{2}}+\frac{1}{2 !} \xi_{1}^{2} \frac{\partial^{2} f(\boldsymbol{x})}{\partial x_{1}^{2}} \\
& +\frac{1}{2 !} \xi_{2}^{2} \frac{\partial^{2} f(\boldsymbol{x})}{\partial x_{2}^{2}}+\xi_{1} \xi_{2} \frac{\partial^{2} f(\boldsymbol{x})}{\partial x_{1} \partial x_{2}},
\end{aligned}
$$

where $\xi_{1}$ and $\xi_{2}$ are the distance between the initial positions of points $k$ and $j$ in the $x_{1}$-axis and $x_{2}$-axis direction, respectively.

The explicit form in PD for variable and its derivatives are defined as:

$$
\left[\begin{array}{c}
f(\boldsymbol{x}) \\
\frac{\partial f(\boldsymbol{x})}{\partial x_{1}} \\
\frac{\partial f(\boldsymbol{x})}{\partial x_{2}} \\
\frac{\partial^{2} f(\boldsymbol{x})}{\partial x_{1}^{2}} \\
\frac{\partial^{2} f(\boldsymbol{x})}{\partial x_{2}^{2}} \\
\frac{\partial^{2} f(\boldsymbol{x})}{\partial x_{1} \partial x_{2}}
\end{array}\right]=\int_{H_{\boldsymbol{x}}} \mu(\boldsymbol{\xi}, t) f(\boldsymbol{x}+\boldsymbol{\xi})\left[\begin{array}{c}
g_{2}^{00}(\boldsymbol{\xi}) \\
g_{2}^{10}(\boldsymbol{\xi}) \\
g_{2}^{01}(\boldsymbol{\xi}) \\
g_{2}^{20}(\boldsymbol{\xi}) \\
g_{2}^{02}(\boldsymbol{\xi}) \\
g_{2}^{11}(\boldsymbol{\xi})
\end{array}\right] d V
$$

where $g_{2}^{p_{1} p_{2}}$ and $\mu(\boldsymbol{\xi}, t)$ are the PD function and state of bond interaction, respectively. The PD function is represented by unknown coefficients $a_{i j}^{p_{1}} p_{2}$ and weighted function $\omega(|\boldsymbol{\xi}|)$ in Eq. (18). The PD function possessed the orthogonality property is expressed as polynomials:

$$
\begin{gathered}
g_{2}^{p_{1} p_{2}}(\boldsymbol{\xi})=a_{00}^{p_{1} p_{2}} \omega(|\boldsymbol{\xi}|)+a_{10}^{p_{1} p_{2}} \omega(|\boldsymbol{\xi}|) \xi_{1}+a_{01}^{p_{1} p_{2}} \omega(|\boldsymbol{\xi}|) \xi_{2} \\
+a_{20}^{p_{1} p_{2}} \omega(|\boldsymbol{\xi}|) \xi_{1}^{2}+a_{02}^{p_{1} p_{2}} \omega(|\boldsymbol{\xi}|) \xi_{2}^{2}+a_{11}^{p_{1} p_{2}} \omega(|\boldsymbol{\xi}|) \xi_{1} \xi_{2}, \\
\omega(|\boldsymbol{\xi}|)=e^{-(2|\boldsymbol{\xi}| / \delta)^{2}}, \\
\boldsymbol{a}=\left[\begin{array}{ccccccc}
a_{00}^{00} & a_{00}^{10} & a_{00}^{01} & a_{00}^{20} & a_{00}^{02} & a_{00}^{11} \\
a_{10}^{00} & a_{10}^{10} & a_{10}^{01} & a_{10}^{20} & a_{10}^{02} & a_{10}^{11} \\
a_{01}^{00} & a_{01}^{10} & a_{01}^{01} & a_{01}^{20} & a_{01}^{02} & a_{01}^{11} \\
a_{20}^{00} & a_{20}^{10} & a_{20}^{01} & a_{20}^{20} & a_{20}^{02} & a_{20}^{11} \\
a_{02}^{00} & a_{02}^{10} & a_{02}^{01} & a_{02}^{20} & a_{02}^{02} & a_{02}^{11} \\
a_{11}^{00} & a_{11}^{10} & a_{11}^{01} & a_{11}^{20} & a_{11}^{02} & a_{11}^{11}
\end{array}\right] .
\end{gathered}
$$


Due to the orthogonality property of the PD functions, it requires to satisfy $\boldsymbol{A} \boldsymbol{a}=\boldsymbol{b} . \boldsymbol{A}$ is the PD shape matrix, $\boldsymbol{b}$ is the matrix of known coefficients, and $\boldsymbol{a}$ is the matrix of unknown coefficients. The matrix of unknown coefficients $\boldsymbol{a}$ is solved by Eq. (23), and the coefficients are substituted into Eq. (18) to obtain the PD functions. The PDDO employs the concept of nonlocal interactions within the horizon in Eq. (17). Therefore, in contrast to CCM, the PDDO possesses the nonlocal characteristics to evaluate field variables and their derivatives without performing any differential.

$$
\begin{aligned}
\boldsymbol{A}=\int_{H_{\boldsymbol{x}}} \mu(\boldsymbol{\xi}, t) \omega(|\boldsymbol{\xi}|) & {\left[\begin{array}{cccccc}
1 & \xi_{1} & \xi_{2} & \xi_{1}^{2} & \xi_{2}^{2} & \xi_{1} \xi_{2} \\
\xi_{1} & \xi_{1}^{2} & \xi_{1} \xi_{2} & \xi_{1}^{3} & \xi_{1} \xi_{2}^{2} & \xi_{1}^{2} \xi_{2} \\
\xi_{2} & \xi_{1} \xi_{2} & \xi_{2}^{2} & \xi_{1}^{2} \xi_{2} & \xi_{2}^{3} & \xi_{1} \xi_{2}^{2} \\
\xi_{1}^{2} & \xi_{1}^{3} & \xi_{1}^{2} \xi_{2} & \xi_{1}^{4} & \xi_{1}^{2} \xi_{2}^{2} & \xi_{1}^{3} \xi_{2} \\
\xi_{2}^{2} & \xi_{1} \xi_{2}^{2} & \xi_{2}^{3} & \xi_{1}^{2} \xi_{2}^{2} & \xi_{2}^{4} & \xi_{1} \xi_{2}^{3} \\
\xi_{1} \xi_{2} & \xi_{1}^{2} \xi_{2} & \xi_{1} \xi_{2}^{2} & \xi_{1}^{3} \xi_{2} & \xi_{1} \xi_{2}^{3} & \xi_{1}^{2} \xi_{2}^{2}
\end{array}\right] d V } \\
\boldsymbol{b} & =\left[\begin{array}{cccccc}
1 & 0 & 0 & 0 & 0 & 0 \\
0 & 1 & 0 & 0 & 0 & 0 \\
0 & 0 & 1 & 0 & 0 & 0 \\
0 & 0 & 0 & 2 & 0 & 0 \\
0 & 0 & 0 & 0 & 2 & 0 \\
0 & 0 & 0 & 0 & 0 & 1
\end{array}\right] \\
& \boldsymbol{a}=\boldsymbol{A}^{-1} \boldsymbol{b} .
\end{aligned}
$$

\subsection{Interaction integral}

The domain form of the interaction integral [21] is employed to extract mixed-mode SIFs. The integral domain $S$ is chosen between $R_{1}$ and $R_{2}$, and the schematic illustration of the interaction integral is given in Fig. 4. Meanwhile, the linear weight function $q$ in Eq. (24) is adopted. In the interaction integral, two states of field quantities that correspond to the actual and auxiliary states are considered. The superscript "aux" denotes an auxiliary state chosen as the asymptotic field near the crack tip. The displacement and stress components of the auxiliary state are taken from Ref. [25]. The crack faces are assumed to be traction-free. The traction-free BCs are enforced along the upper and lower surfaces of the crack [20]. 


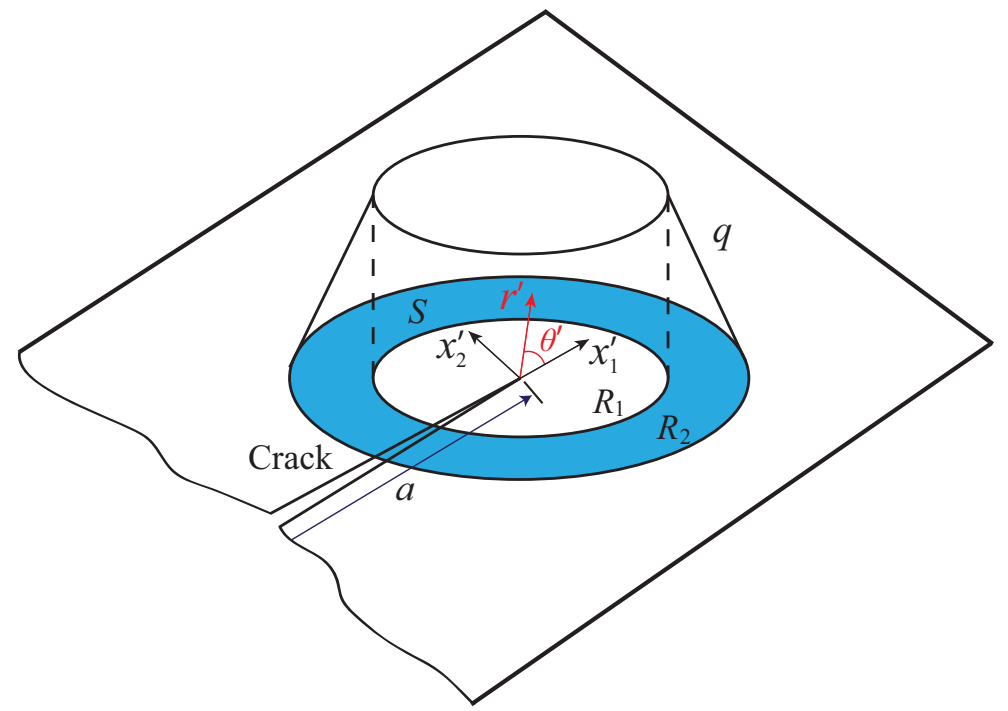

Figure 4: Weight function $q$ for the interaction integral.

$$
q= \begin{cases}1 & \text { on } R_{1} \\ 0 & \text { on } R_{2} \\ 0<q<1 & R_{2}>r^{\prime}>R_{1}\end{cases}
$$

The domain form of the interaction integral $I$ is written as:

$$
I=\int_{S}\left\{\left[\sigma_{i j} u_{i, 1}^{a u x}+\sigma_{i j}^{a u x} u_{i, 1}\right]-W^{i n t} \delta_{1 j}\right\} q_{, j} d S,
$$

where $\delta_{i j}$ and $W^{\text {int }}$ denote the Kronecker delta property and interaction strain energy, respectively. $W^{\text {int }}$ is defined as below:

$$
W^{i n t}=\sigma_{i j} \varepsilon_{i j}^{a u x}=\sigma_{i j}^{a u x} \varepsilon_{i j} .
$$

The interaction integral $I$ is written in terms of SIFs in Eq. (27). $K_{I}$ and $K_{I I}$ can be evaluated by choosing appropriate auxiliary fields. While the auxiliary field of mode-I $\left(K_{I}^{a u x}=1\right.$ and $\left.K_{I I}^{a u x}=0\right)$ is selected, $K_{I}$ is obtained 
from Eq. (27). $K_{I I}$ can be extracted in a similar fashion by selecting the auxiliary field of mode-II ( $K_{I}^{a u x}=0$ and $\left.K_{I I}^{a u x}=1\right)$.

$$
I=\frac{2}{E}\left[K_{I} K_{I}^{a u x}+K_{I I} K_{I I}^{a u x}\right]
$$

\section{Numerical examples}

Four numerical examples for single- and mixed-mode fracture problems are considered to examine the accuracy and effectiveness of the proposed approach. Meanwhile, it aims to confirm the performance of the PD framework in linear elastic fracture mechanics (LEFM). All the PD results are compared with reference results or exact solutions. Please note that in-plane loads are only considered in the present paper. Additionally, uniform particle distribution is taken in the following examples. The material particle spacing is denoted as $\Delta x$. All the examples of shell thickness $h$ and horizon size $\delta$ are set to 1 unit and 3.015 $\Delta x$, respectively. The normalized SIFs are defined as $F_{i}=K_{i} / \sigma \sqrt{\pi a}$, where $i=I$ for mode-I and $i=I I$ for mode-II.

\subsection{Flat square shell with an edge crack under tensile load}

A flat square shell including an edge crack under tensile load is analyzed. The geometry is shown in Fig. 5 (a). The length $L$ and width $W$ are $100 \mathrm{~mm}$, and the crack length is $a=50 \mathrm{~mm}$. $E=72 \mathrm{GPa}$ and $\nu=1 / 3$ are chosen for the material properties. The uniform tensile stress $\sigma=1.0 \mathrm{MPa}$ is applied to the top and bottom edges. Four different particle spacings are taken, including $\Delta x=2.0,1.0,0.5$, and $0.4 \mathrm{~mm}$.

SIF represents a "stress state" near the crack tip for providing a quantity to describe failure behaviors in fracture mechanics. In order to examine the ability of the proposed approach, the stress components of $\sigma_{11}$ and $\sigma_{22}$ are compared between PD and FEM in the first example. As shown in Figs. 6 and $7, \sigma_{11}$ and $\sigma_{22}$ distributions in PD are almost the same as the FEM results. Moreover, $\sigma_{22}$ distribution along the $r^{\prime}$-axis near the crack tip is investigated with and without the energy method when the $\theta^{\prime}$-axis equals $0^{\circ}$. From the comparison in Fig. 8 (a), the results with the energy method is more close to the FEM distribution than the results without the energy method. It is found that the energy method can effectively correct the surface effect in analyzing stress near the crack. Thus, the energy method is employed in the following examples. 
(a)

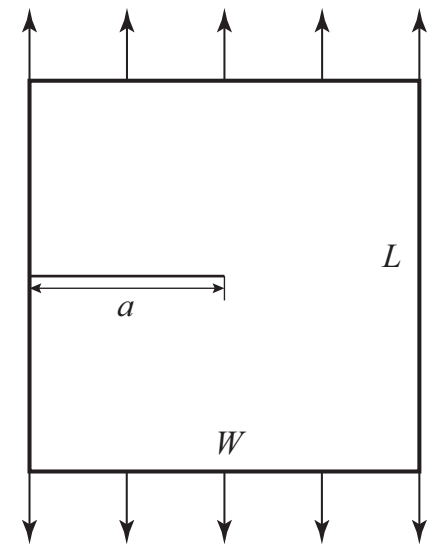

(b)

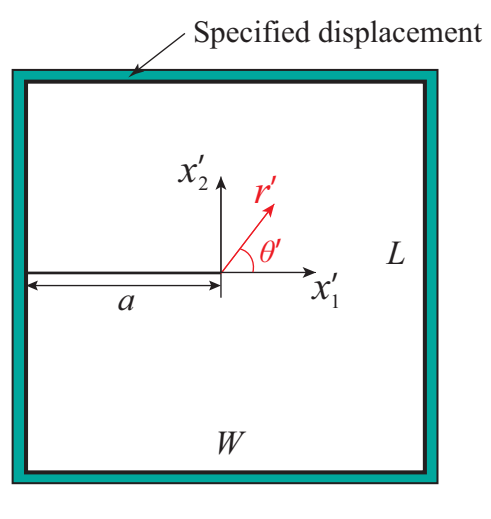

Figure 5: Flat square shell with an edge crack, (a) Under tensile load, (b) Under specified displacement BCs.

(a)

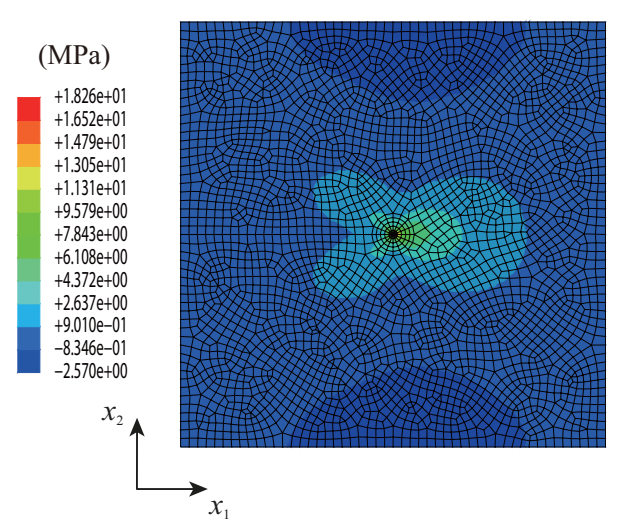

(b)

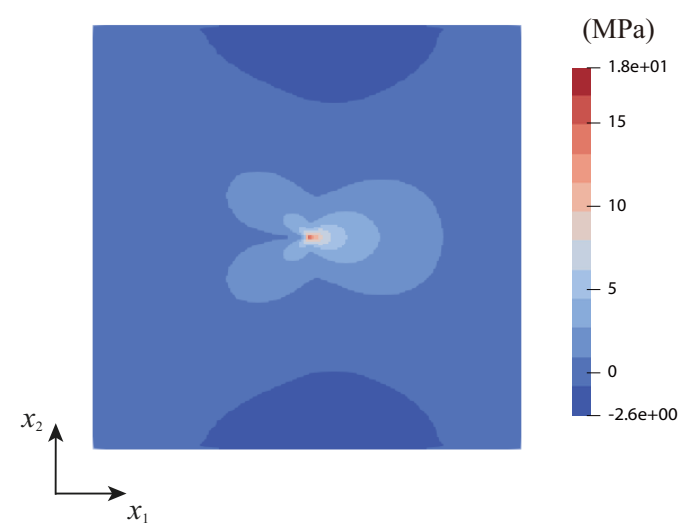

Figure 6: Comparison of $\sigma_{11}$ between FEM and PD, (a) FEM, (b) PD. 
(a)

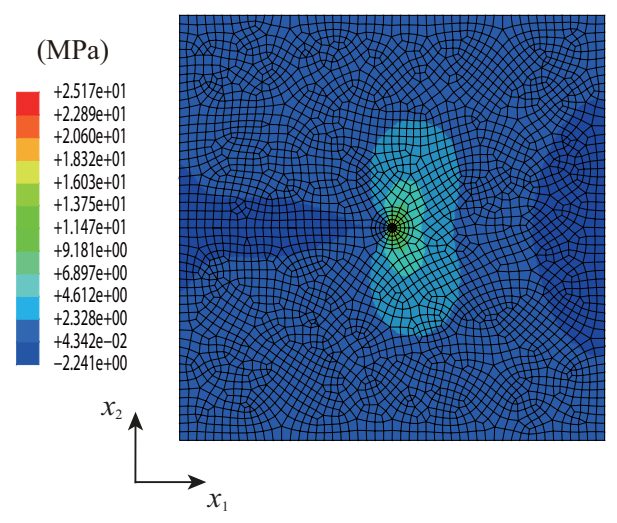

(b)

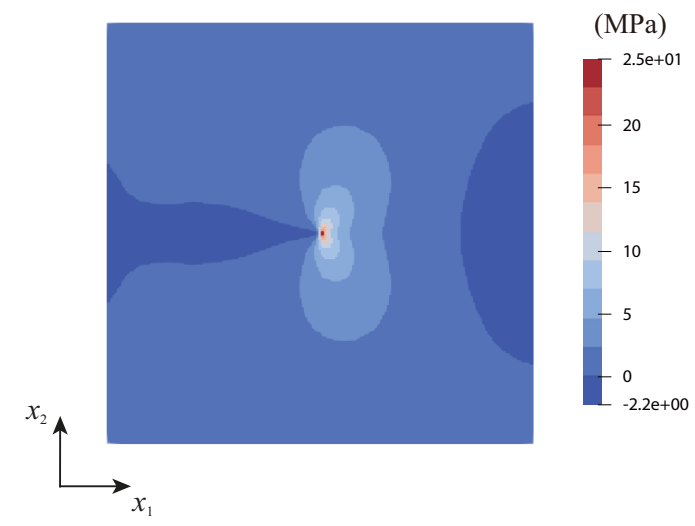

Figure 7: Comparison of $\sigma_{22}$ between FEM and PD, (a) FEM, (b) PD.

(a)

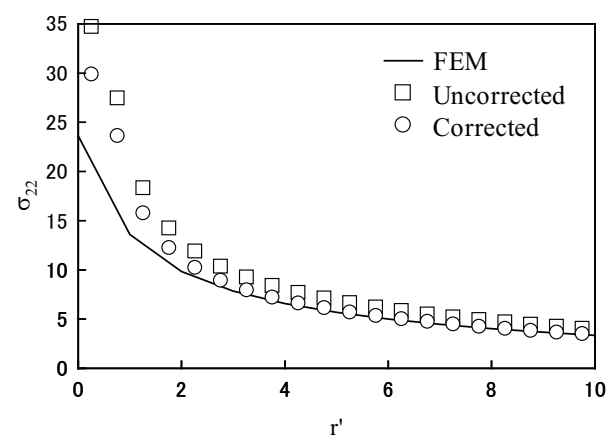

(b)

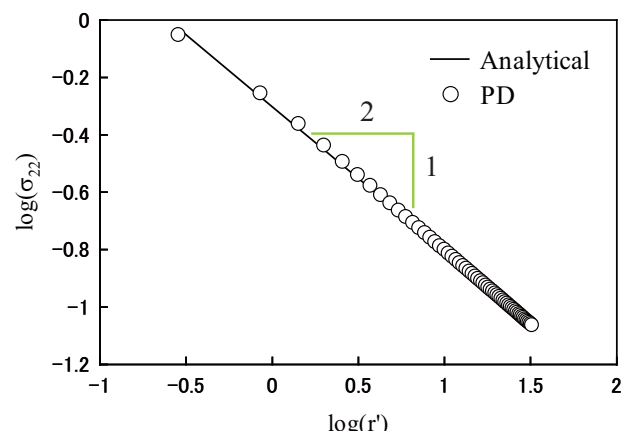

Figure 8: (a) $\sigma_{22}$ distribution along the $r^{\prime}$-axis with and without the energy correction method under tensile load when the $\theta^{\prime}$-axis equals $0^{\circ}$, (b) $\log \left(\sigma_{22}\right)$ distribution along the $r^{\prime}$-axis under specified displacement BCs when the $\theta^{\prime}$-axis equals $45^{\circ}$. 
To further investigate the effectiveness of capturing stress fields near the crack tip, the specified displacement BCs corresponding to the $K$-field solution [26] is adopted, as illustrated in Fig. 5 (b). For this additional investigation, $E=1 \mathrm{GPa}$ and $\nu=0.25$ are assumed. The specified displacement BCs are expressed in Eq. (28) as:

$$
\left[\begin{array}{l}
u \\
v
\end{array}\right]=\frac{K_{I}}{2 G} \sqrt{\frac{r^{\prime}}{2 \pi}}\left[\begin{array}{c}
\cos \frac{\theta^{\prime}}{2}\left(\kappa-1+2 \sin ^{2} \frac{\theta^{\prime}}{2}\right) \\
\sin \frac{\theta^{\prime}}{2}\left(\kappa+1-2 \cos ^{2} \frac{\theta^{\prime}}{2}\right)
\end{array}\right],
$$

where $G=E / 2(1+\nu)$ and $\kappa=(3-\nu) /(1+\nu) . \quad K_{I}=1.0 \mathrm{MPa} \sqrt{\mathrm{mm}}$ is prescribed. The polar coordinates $\left(r^{\prime}, \theta^{\prime}\right)$ are defined at the crack tip.

The stress component $\sigma_{22}$ is calculated along the $r^{\prime}$-axis under the specified displacement $\mathrm{BCs}$ when the $\theta^{\prime}$-axis equals $45^{\circ} \cdot \log \left(\sigma_{22}\right)$ distribution near the crack tip is given in Fig. $8(\mathrm{~b})$. The slope of $\log \left(\sigma_{22}\right)$ equals 0.5 that satisfy the physical behavior in LEFM, and the results have a good agreement with the analytical results from the $K$-field solution [26]. From the results mentioned above, it is demonstrated that the stress fields in LEFM can be captured by the proposed method.

The convergence analysis of $K_{I}$ is carried out in Fig. 9. The reference result of $K_{I}$ in this example is $37.72 \mathrm{MPa} \sqrt{\mathrm{mm}}$ computed from the J-integral value in Ref. [13]. While the particle spacing is less than $0.5 \mathrm{~mm}$, the error of $K_{I}$ is less than $1 \%$. At the same time, the path-independence analysis of $K_{I}$ is implemented with four different horizon sizes, including $\delta=6.0,3.0$, 1.5 , and $1.2 \mathrm{~mm}$. According to Ref. [18], it recommends that the parameters of the interaction integral $R_{2}-R_{1}$ and $R_{1}$ are set larger than $\delta$ to reduce the influence of the surface effect. From Table 1, the path-independence of $K_{I}$ can be found. While the ratio of $\left(R_{2}-R_{1}\right) / a$ is greater than or equal to 0.2 , the steady PD results are obtained.

Table 1: SIF for different horizon sizes and integral domains.

\begin{tabular}{ccccccc}
\hline & \multicolumn{6}{c}{$\left(R_{2}-R_{1}\right) / a$} \\
\cline { 2 - 7 }$\delta$ & 0.1 & 0.2 & 0.3 & 0.4 & 0.5 & 0.6 \\
\hline $6.0 \mathrm{~mm}$ & 36.467 & 38.710 & 38.229 & 39.160 & 38.571 & 39.056 \\
$3.0 \mathrm{~mm}$ & 38.830 & 38.444 & 38.471 & 38.300 & 38.334 & 38.309 \\
$1.5 \mathrm{~mm}$ & 37.509 & 38.006 & 37.925 & 37.949 & 38.006 & 38.058 \\
$1.2 \mathrm{~mm}$ & 37.619 & 37.702 & 37.785 & 37.767 & 37.771 & 37.808 \\
\hline
\end{tabular}




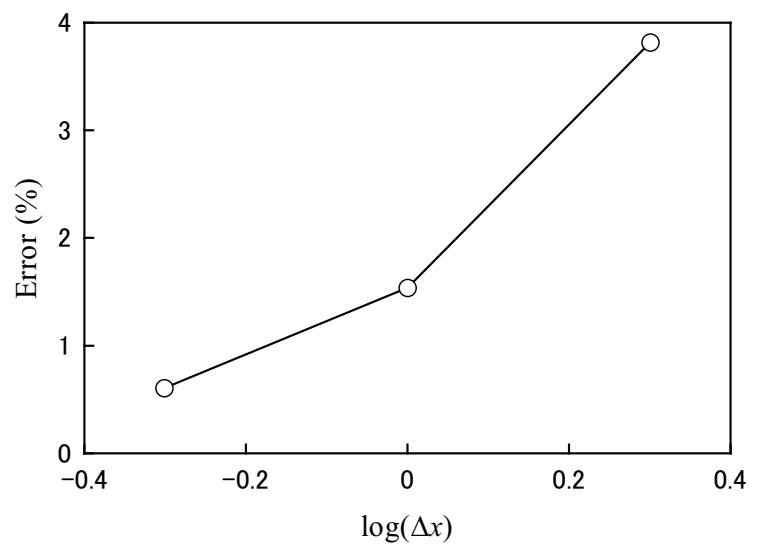

Figure 9: Convergence analysis of $K_{I}$.

\subsection{Flat rectangular shell with an inclined edge crack under tensile load}

A flat rectangular shell including an inclined edge crack under tensile load is considered. The length $L$ and width $W$ of the rectangular shell are $20 \mathrm{~mm}$ and $10 \mathrm{~mm}$, respectively. Four crack lengths $a / W$ are set from 0.2 to 0.5 , and two crack angles $\beta=30^{\circ}$ and $45^{\circ}$ are taken. The uniform tensile stress $\sigma=1.0 \mathrm{MPa}$ is chosen. The analysis model is illustrated in Fig. 10. Young's modulus $E$ and Poisson's ratio $\nu$ are $206 \mathrm{GPa}$ and 0.3 , respectively. Three different particle spacings $\Delta x=0.125,0.0625$, and $0.03125 \mathrm{~mm}$ are adopted.

This example is a mixed-mode fracture problem. The convergence analysis is carried out for mode-I and -II SIFs. From Fig. 11, it can be clearly found that the error of $K_{I}$ is obviously less than the error of $K_{I I}$ with the same number of discretization nodes. In this example, it is recommended that the particle spacing is less than or equal to $0.03125 \mathrm{~mm}$.

Additionally, the normalized mixed-mode SIFs for different crack lengths and crack angles are investigated. In order to examine the accuracy of the proposed method, the reference results are chosen from the meshfree method [27] and the mapping technique [28]. The comparisons between three different methods are presented in Fig. 12. From the comparisons, the PD results of $\beta=30^{\circ}$ and $\beta=45^{\circ}$ agree well with the other two reference results. 


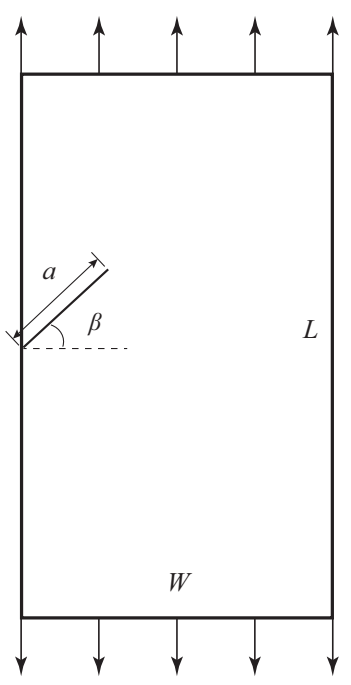

Figure 10: Flat rectangular shell under tensile load with an inclined edge crack.

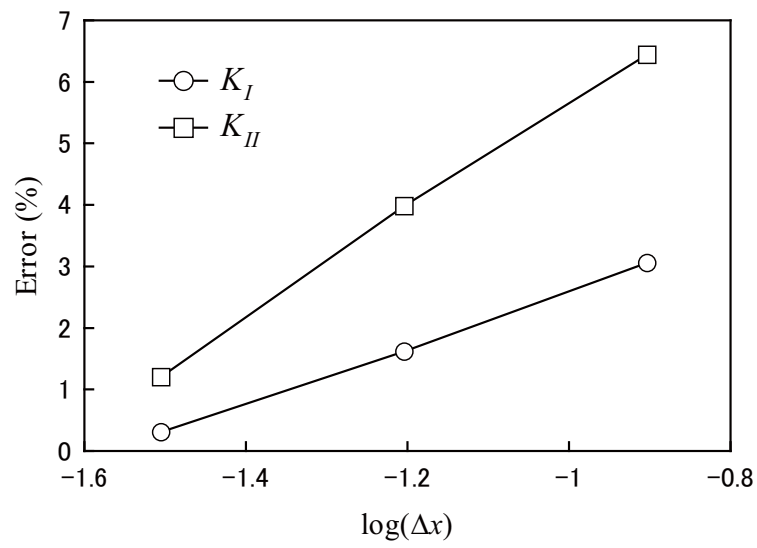

Figure 11: Convergence analysis of $K_{I}$ and $K_{I I}$. 
(a)

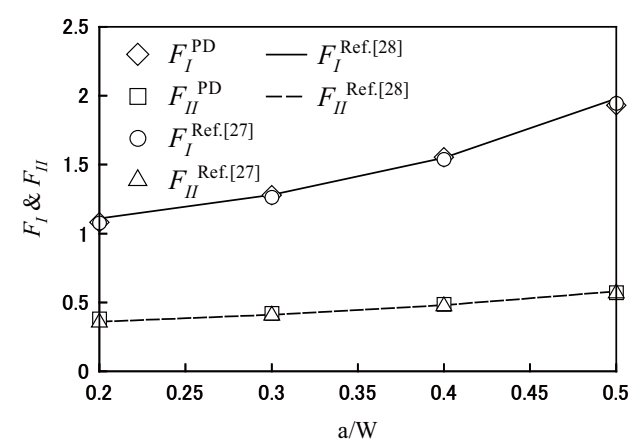

(b)

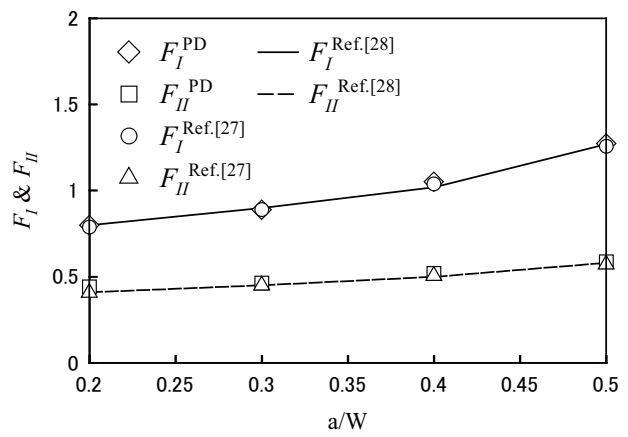

Figure 12: Normalized mixed-mode SIFs for different crack lengths, (a) $\beta=30^{\circ}$, (b) $\beta=45^{\circ}$.

\subsection{Flat square shell with an inclined central crack under tensile load}

A flat square shell including an inclined central crack under tensile load is investigated. The geometry sizes are $L=W=10 \mathrm{~mm}$. The material properties of this example are the same as the second example. The shell is subjected to uniaxial stress, $\sigma=1.0 \mathrm{MPa}$, as shown in Fig. 13. The crack lengths $2 a / W$ are adopted from 0.1 to 0.7 , and the crack angle $\beta$ is varied from $0^{\circ}$ to $90^{\circ}$. The particle spacing $\Delta x=0.03125 \mathrm{~mm}$ is used.

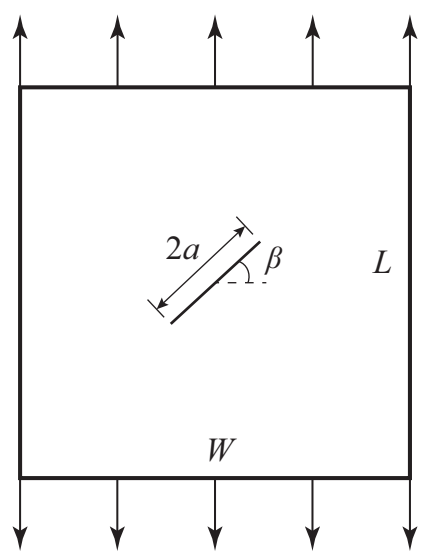

Figure 13: Flat square shell under tensile load with an inclined center crack.

First, the normalized mode-I SIF is evaluated for different crack lengths 
when $\beta$ equals $0^{\circ}$, and the results are compared with Ref. [29]. From Fig. 14 (a), the PD results have a good agreement with the reference results. Meanwhile, the normalized SIF approaches 1.0 when $2 a / W$ equals 0.1 . It means that the case of $2 a / W=0.1$ can regard as an infinite plate under farfield uniaxial stress.

Next, the normalized mixed-mode SIFs for different crack angles are calculated. According to the conclusion mentioned above, the analysis model can be considered as an infinite plate subjected to far-field uniaxial stress when $2 a / W=0.1$. Therefore, the exact solutions in Eq. (29) can be taken to compare with the PD results. Meantime, the XFEM results from Ref. [21] are also adopted to be reference results. The results from three different approaches are illustrated in Fig. 14 (b). Even though the crack length is much less than the shell width, the analysis model is not an infinite plate. Thus, the PD results are more close to the XFEM results instead of the exact solutions. In conclusion, the PD results have good accuracy by comparing three different approaches.

$$
K_{I}=\sigma \sqrt{\pi a} \cos ^{2} \beta, \quad K_{I I}=\sigma \sqrt{\pi a} \cos \beta \sin \beta .
$$

(a)

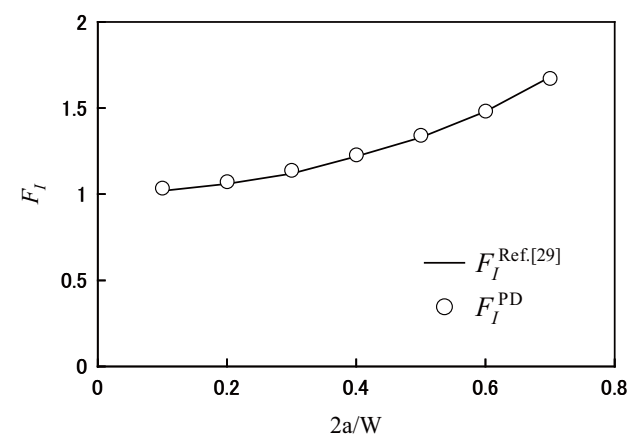

(b)

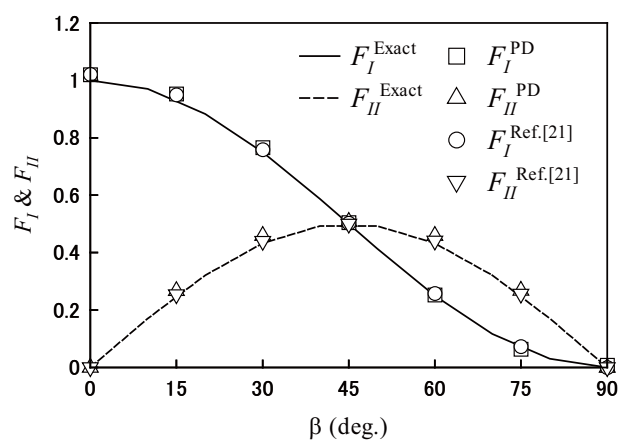

Figure 14: (a) Normalized SIF for different crack lengths at $\beta=0^{\circ}$, (b) Normalized mixedmode SIFs for different crack angles. 
4.4. Flat rectangular shell with an inclined eccentric crack under tensile load

The last numerical example deals with a flat rectangular shell under tensile load including an inclined eccentric crack. The geometry sizes, tensile load, and material properties of this example are identical to the second example, whereas the half crack length and crack angle have different arrangements. The half crack length $a$ is fixed at $1 \mathrm{~mm}$, and $e=W / 4$ is a distance for the crack position away from the center of the flat shell. Seven crack angles $\beta$ are considered, including $0^{\circ}, 15^{\circ}, 30^{\circ}, 45^{\circ}, 60^{\circ}, 75^{\circ}$, and $90^{\circ}$. The geometry and crack position are given in Fig. 15. In this example, the particle spacing $\Delta x=0.04 \mathrm{~mm}$ is set.

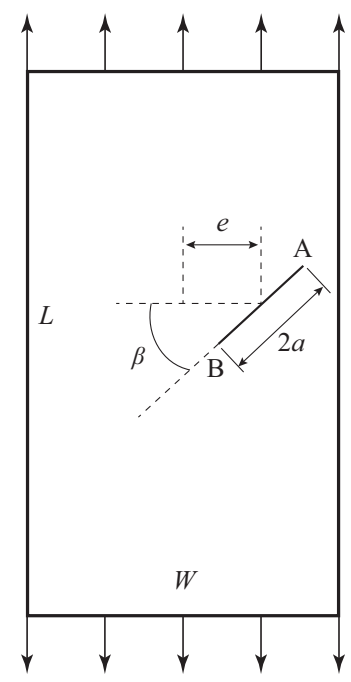

Figure 15: Flat rectangular shell under tensile load with an inclined eccentric crack.

The normalized mixed-mode SIFs of eccentric crack for different crack angles are assessed. Owing to the crack position which does not locate at the center of the shell, different SIF results are found for each crack tip. The PD results are compared with the results from FEM software, Abaqus [30]. The comparisons of crack tip A and B are presented in Fig. 16 (a) and (b), respectively. The PD results of $K_{I}$ and $K_{I I}$ for each crack tip closely approach the FEM results. Only eliminating interaction bonds is used to model the inclined eccentric crack without special numerical treatments. The advantage of crack modeling in the PD theory is demonstrated in this example. 
(a)

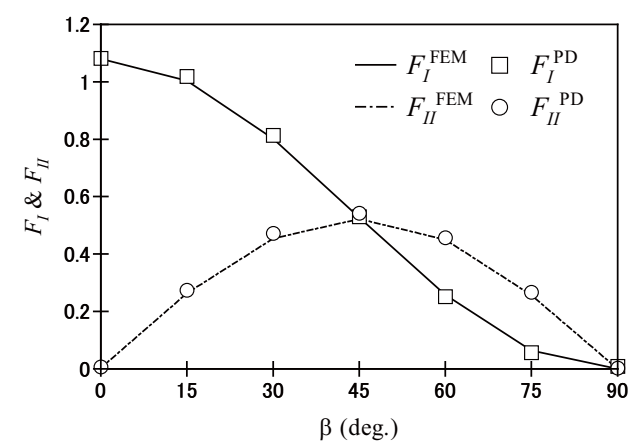

(b)

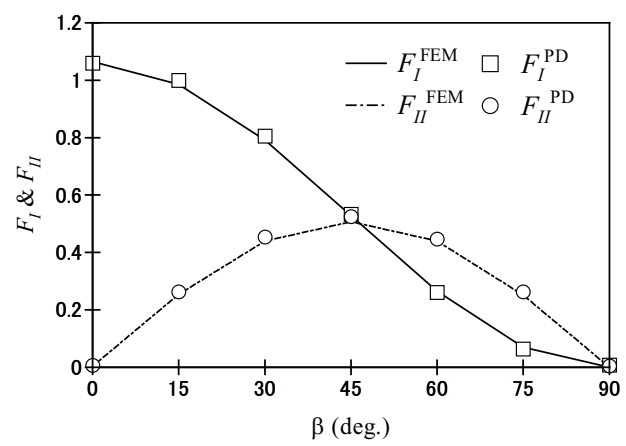

Figure 16: Normalized mixed-mode SIFs for different crack angels, (a) Crack tip A, (b) Crack tip B.

\section{Conclusion}

The mixed-mode SIFs of flat shells under in-plane loading are evaluated in the OSPD framework. The PDDO is adopted to calculate strain and stress components, and those components are substituted into the interaction integral to evaluate SIFs. Meanwhile, the energy method is used to reduce the influence of the surface effect. To obtain steady-state solutions, the ADR technique is employed for static problems. The accuracy and effectiveness of the proposed approach are examined by comparing PD results with exact solutions and other numerical methods. According to the comparisons in the numerical examples, the mixed-mode SIFs are accurately evaluated. Moreover, the advantage of crack modeling in the PD theory is also demonstrated by eliminating interaction bonds. The arbitrary crack can be easily modeled without special numerical treatments. The proposed PD approach achieves the performance of LEFM and provides an alternative approach for mixedmode SIFs evaluation under in-plane loading. The research objectives of future work are to evaluate fracture parameters under out-of-plane loading.

\section{Acknowledgements}

This research was partially supported by Sasakawa Scientific Research Grant from the Japan Science Society. 


\section{References}

[1] S.A. Silling, Reformulation of elasticity theory for discontinuities and long-range forces, J. Mech. Phys. Solids. 48 (2000) 175-209.

[2] S.A. Silling, M. Epton, O. Weckner, J. Xu, E. Askari, Peridynamic states and constitutive modeling, J. Elast. 88 (2007) 151-184.

[3] J. O'Grady, J. Foster, Peridynamic plates and flat shells: A nonordinary, state-based model, Int. J. Solids Struct. 51 (2014) 4572-4579.

[4] C. Diyaroglu, E. Oterkus, S. Oterkus, E. Madenci, Peridynamics for bending of beams and plates with transverse shear deformation, Int. J. Solids Struct. 69 (2015) 152-168.

[5] S.R. Chowdhury, P. Roy, D. Roy, J.N. Reddy, A peridynamic theory for linear elastic shells, Int. J. Solids Struct. 84 (2016) 110-132.

[6] C.T. Nguyen, S. Oterkus, Peridynamics for the thermomechanical behavior of shell structures, Eng. Fract. Mech. 219 (2019) 106623.

[7] Z. Yang, B. Vazic, C. Diyaroglu, E. Oterkus, S. Oterkus, A Kirchhoff plate formulation in a state-based peridynamic framework, Math. Mech. Solids. 25 (2020) 727-738.

[8] C.T. Nguyen, S. Oterkus, Investigating the effect of brittle crack propagation on the strength of ship structures by using peridynamics, Ocean Eng. 209 (2020) 107472.

[9] S.A. Silling, E. Askari, A meshfree method based on the peridynamic model of solid mechanics, Comput. Struct. 83 (2005) 1526-1535.

[10] J.T. Foster, S.A. Silling, W. Chen, An energy based failure criterion for use with peridynamic states, J. Multiscale. Struct. Eng. 9 (2011) 675-687.

[11] E. Madenci, S. Oterkus, Ordinary state-based peridynamics for plastic deformation according to von Mises yield criteria with isotropic hardening, J. Mech. Phys. Solids. 86 (2016) 192-219. 
[12] D. Dipasquale, G. Sarego, M. Zaccariotto, U. Galvanetto, A discussion on failure criteria for ordinary state-based peridynamics, Eng. Fract. Mech. 186 (2017) 378-398.

[13] W. Hu, Y.D. Ha, F. Bobaru, S.A. Silling, The formulation and computation of the nonlocal J-integral in bond-based peridynamics, Int. J. Fract. 176 (2012) 195-206.

[14] M.S. Breitenfeld, P.H. Geubelle, O. Weckner, S.A. Silling, Nonordinary state-based peridynamic analysis of stationary crack problems, Comput. Methods Appl. Mech. Engrg. 272 (2014) 233-250.

[15] Q.V. Le, F. Bobaru, Surface corrections for peridynamic models in elasticity and fracture, Comput. Mech. 61 (2018) 499-518.

[16] C. Stenström, K. Eriksson, The J-contour integral in peridynamics via displacements, Int. J. Fract. 216 (2019) 173-183.

[17] J. Jung, J. Seok, Mixed-mode fatigue crack growth analysis using peridynamic approach, Int. J. Fatigue 103 (2017) 591-603.

[18] M. Imachi, S. Tanaka, T.Q. Bui, Mixed-mode dynamic stress intensity factors evaluation using ordinary state-based peridynamics, Theor. Appl. Fract. Mech. 93 (2018) 97-104.

[19] E. Madenci, E. Oterkus, Peridynamic theory and its applications, Springer, New York, 2014.

[20] E. Madenci, A. Barut, M. Futch, Peridynamic differential operator and its applications, Comput. Methods Appl. Mech. Engrg. 304 (2016) 408451.

[21] N. Moës, J. Dolbow, T. Belytschko, A finite element method for crack growth without remeshing, Int. J. Numer. Meth. Eng. 46 (1999) 131150 .

[22] T. Dirgantara, M.H. Aliabadi, Stress intensity factors for cracks in thin plates, Eng. Fract. Mech. 69 (2002) 1465-1486.

[23] S. Tanaka, M.J. Dai, S. Sadamoto, T.T. Yu, T.Q. Bui, Stress resultant intensity factors evaluation of cracked folded structures by 6 DOFs flat shell meshfree modeling, Thin-Walled Struct. 144 (2019) 106285. 
[24] S.A. Silling, R.B. Lehoucq, Convergence of peridynamics to classical elasticity theory, J. Elasticity. 93 (2008) 13-37.

[25] M. Malekan, F.B. Barros, Numerical analysis of a main crack interactions with micro-defects/inhomogeneities using two-scale generalized/extended finite element method, Comput. Mech. 62 (2018) 783801.

[26] T.L. Anderson, Fracture mechanics: fundamentals and applications, CRC Press, 2004.

[27] S. Tanaka, H Suzuki, S. Sadamoto, S. Sannomaru, T.T. Yu, T.Q. Bui, J-integral evaluation for 2D mixed-mode crack problems employing a meshfree stabilized conforming nodal integration method, Comput. Mech. 58 (2016) 185-198.

[28] O.L. Bowie, Solutions of plane crack problems by mapping technique, Methods of Analysis and Solutions of Crack Problems, G. C. Sih, ed., Noordhoff International Publishing, Leyden, 1973.

[29] M. Isida, Effect of width and length on stress intensity factors of internally cracked plates under various boundary conditions, Int. J. Fract. 7 (1971) 301-316.

[30] Dassault Systemes, Simulia, Abaqus 6.13 User Manual, Providence, RI, USA. 2013. 